\title{
Dunkelziffer der Bequemlichkeit
}

\section{Bernhard Gurtner}

Für einmal bitte ganz ehrlich: Warum sind Sie Augenärztin geworden? Oder Neurochirurg?

Anästhesistin? Radiologe? Hausärztin in einer Gruppenpraxis? Welche Faktoren beeinflussten ihre glatte oder holprige medizinische Laufbahn? Gelang alles nach Plan, oder haben zufällige Ereignisse zum Kurswechsel nach Betätigung der Notbremse geführt? Sind Sie etwa aus Unachtsamkeit oder Bequemlichkeit in einem Zug sitzen geblieben, der Sie an ein Ziel gebracht hat, das Sie gar nicht erreichen wollten?

Es war bequemer, mehrere Jahre an der gleichen Klinik $\mathrm{zu}$ verbleiben, als immer wieder an einen neuen Arbeitsort zu wechseln, wo man im Ärzterudel zuhinterst anzustehen hat und sich in einem ungewohnten Klinikalltag und Sprachgebrauch zurechtfinden muss. Verblieben deshalb so viele in jenem Spezialgebiet, das ihnen nach dem Staatsexamen als Erstes zur Weiterbildung offenstand? Nicht selten wandelte ein eigennütziger Chefarzt die für zukünftige Allgemeinpraktiker reservierte Rotationsstelle in ein Facharzt-Curriculum um. Eine Win-win-Strategie: Der tüchtige und bereits gut eingeführte Mitarbeiter blieb der Klinik erhalten; der Assistent musste keinen anderen Wohnort suchen, durfte die Freundin und das gleiche Autonummernschild behalten und konnte sich auf mehr Prestige und Einkommen nach der FMH-Diplomierung freuen.

Für das Überangebot an Spezialisten in den Zentren und den allgemeinen Mangel an Grundversorgern haben solche beidseits bequemen Weiterbildungskorrekturen nicht unwesentlich beigetragen. Potentielle Grundversorger wurden abgeworben. Als Dunkelziffer fehlen sie in den Statistiken der inneren und äusseren Beweggründe, welche zum definitiven Berufsziel leiten.

$\mathrm{Zu}$ Beginn des Medizinstudiums wissen nur wenige ganz genau, welche Fachrichtung sie wählen werden. Vorgespurt ist die Laufbahn, wenn Sprösslinge einer Ärztefamilie die Praxis des Vaters oder eines Onkels übernehmen dürfensollenwollen. Die anderen Neulinge betreten zunächst einmal staunend das weite Feld der medizinischen Wissenschaften und freuen sich nach Seziersaal und Kommunikationstraining mit Schauspielerpatienten auf Kontakte mit echten Kranken. Nach einigen Semestern wissen sie wenigstens, welche Fächer für sie keinesfalls in Frage kämen. Bis zum Staatsexamen entscheidet sich etwa die Hälfte aller Studienabgänger definitiv, wohin die Weiter- bildung führen soll. Hat Sie ein Professor in den Vorlesungen oder eine Oberärztin im Praktikum für Ihr Fachgebiet begeistert? Waren schliesslich ganz andere Beweggründe und Begleitumstände entscheidend? Die Möglichkeit für Teilzeitarbeit, die Bedürfnisse von Partner oder Familie? Kopf oder Bauch? Haben Sie sich erkundigt, welche Marktnischen bestünden? Gab es unglückliche Zufälle und lästige Umwege, die unerwartet wertvolle zusätzliche Kompetenzen vermittelten? Mir hat erst der Lawinentod eines lieben Kollegen eine Assistentenstelle an einer Universitätsklinik verschafft, in einem Fach, das mich primär nicht interessiert hätte, dann aber faszinierend war.

\section{Bitte ganz ehrlich: Warum sind Sie Augenärztin geworden? Oder Neurochirurg?}

Wenn heute $85 \%$ der Studienabgänger angeben, dass familienfreundliche Arbeitsbedingungen für ihre Fachrichtungswahl mitentscheidend sind*, wäre zu hoffen, dass die Scheidungsrate der Arztehen bald einmal absinken würde. Arbeitszeitverkürzungen schützen aber offensichtlich nicht vor Ehekrisen. Gilt demnach eine maliziöse Behauptung nur für Ärzte, die siebzig Stunden pro Woche mit dem Spital oder ihrer Praxis verheiratet sind?

«Arztehen sind glücklich. Das kommt daher, dass, wenn ein Arzt seine silberne Hochzeit feiert, er mit seiner Frau zwar brutto fünfundzwanzig, aber netto höchstens fünf Jahre verheiratet gewesen ist» (Peter Bamm, Chirurg und Schriftsteller alias Curt Emmrich, 1897-1975).

\section{Anmerkung}

* Dieser Beitrag ist als Ergänzung gedacht zu einer im Auftrag des BAG verfassten Studie zur Steuerung der ärztlichen Weiterbildung in der Schweiz. Büro für Arbeits- und sozialpolitische Studien BASS AG, Bern. www.buerobass.ch/pdf/2014/BAG

SteuerungAerztlicheWB_Schlussbericht.pdf

Die Studie erfasst mit zahlreichen statistischen Daten der letzten zwanzig Jahre den unausgewogenen Ist-Zustand der fachlichen und regionalen Fachärzteverteilung in der Schweiz. Es werden die inneren und äusseren Faktoren dargelegt, die im Studium und in der Weiterbildungszeit zur Wahl des Fachgebietes und des Niederlassungsortes führen. Schliesslich werden Steuerungsmechanismen diskutiert, die einer Über- oder Unterversorgung vorbeugen könnten. Zulassungsstopps haben sich als wirksames Instrument erwiesen, müssten aber durch liberalere Interventionen ersetzt werden, die schon in früheren Phasen der Aus- und Weiterbildung lenkend eingreifen. Hierfür einen akademischen und politischen Konsens zu finden, erscheint momentan fast aussichtslos. Siehe auch: Kesseli B. Ergiebige Gedankenarbeit auf schwierigem Terrain. Schweiz Ärztezeitung. 2015; 96(11):376-80. 